\title{
Effect of stimulus intensity on the rising phase of the nerve action potential in healthy subjects and in patients with peripheral nerve lesions
}

\author{
F. PODIVINSKÝ
} From the Department of Electrophysiology of the Nervous System, Institute of Normal and Pathological
Physiology of the Slovak Academy of Sciences, Bratislava, Czechoslovakia

The nerve action potential is the most usual indicator of the presence of a nerve impulse in a peripheral nerve. The impulses travel along the axons as brief electrical waves. The essential shape characteristics of the nerve action potential (a bior triphasic wave) are determined by the distribution of electric current in a volume conductor during bipolar recording (Lorente de Nó, 1947a, b; Lloyd, 1952; Kostyuk, 1956; and Bureš, Petrán̆, and Zachar, 1960) and will not be considered here. Our earlier papers (Podivinský, 1965a, b) described the effect of stimulus intensity on the amplitude and latency of the nerve action potential in healthy subjects and in patients with a peripheral nerve lesion. Recently, the relation of fibre diameter to the rising and falling phases of the spike was studied in medullated nerve fibres of cats (Paintal, 1966).

In this study, interest was centred mainly on the relationship between stimulus intensity and rate of rise of the potential amplitude. On purely physiological grounds, the rate of rise of a potential seems to be just as important a parameter of the conducted response as is amplitude. The problem raised by the present experiments is whether this type of modification of the nerve action potential can give some information about pathophysiological conditions and so contribute to detecting the early stages of a nerve lesion.

\section{METHODS AND MATERIAL}

The technique employed was that reported by Dawson and Scott (1949) and Gilliatt and Sears (1958). Details of the method used in this study were similar to those previously described by Podivinský (1965a, b).

Bipolar surface electrodes were used for recording and stimulating. Nerve action potentials were recorded from the ulnar nerve, at the elbow, after electrical stimulation at the wrist. As the rise time of the nerve action potential recorded with bipolar electrodes varies according to the separation of the electrodes, for comparative purposes, the interelectrode distance should be maintained constant. In order to ensure a constant separation of $3 \mathrm{~cm}$. surface recording electrodes of the type originally described by Dawson and Scott (1949) were used in this study. Amplified potentials were displayed on one beam of a double beam cathode-ray oscilloscope for photographic recording above a time scale registered on the second oscilloscope beam. Rectangular stimuli of 0.2 and 0.5 msec. duration were delivered at 0.3 and 1.0 per second from an electric stimulator with low output impedance (100 ohms) through an isolating unit.

The steepness of the rising face of the action potential spike (Fig. 1) may be measured as the rise time of the negative (upward) deflection of the triphasic response $(\triangle T)$ or as tangent of an angle between the rising face of the negative phase and the baseline $(\tan \alpha)$. In addition to these parameters the length of rise of the negative potential deflection $\lambda$ can be measured. From a purely physical aspect the rise length $\lambda$ may be defined as the shortest distance between the not yet excited point of the nerve and that of maximum depolarization. It is directly proportional to the conduction velocity (v) and the rise time of the negative deflection of the nerve response $(\triangle T)$, according to equation

$$
\lambda=\mathrm{v} . \triangle \mathbf{T}
$$

The stimulating voltage varied from a threshold value to a supramaximal electric stimulus (up to 150 volts), thus permitting evaluation of the influence of stimulus intensity on the time course of the nerve action potential.

Observations were performed in 50 healthy subjects with an age range of 18 to 51 years and in 25 patients, aged 20 to 55 years, suffering from slight peripheral nerve lesions. In all patients, mild sensory disturbances were present, mainly in the region innervated by the tested ulnar nerve. Ten had painful conditions of the upper limb due to intervertebral disk herniation in the cervical region. Four showed initial damage to the ulnar nerve at the elbow. Three had circumscribed arachnoiditis in the cervical region. In eight patients, the diagnosis was diabetic neuropathy. Investigations were carried out at room temperature, maintained between $22^{\circ} \mathrm{C}$. and $24^{\circ} \mathrm{C}$. The mean skin temperature of the examined limbs in the control group was $31 \cdot 2^{\circ} \mathrm{C}$; in the patients' group it was $30 \cdot 6^{\circ} \mathrm{C}$. 


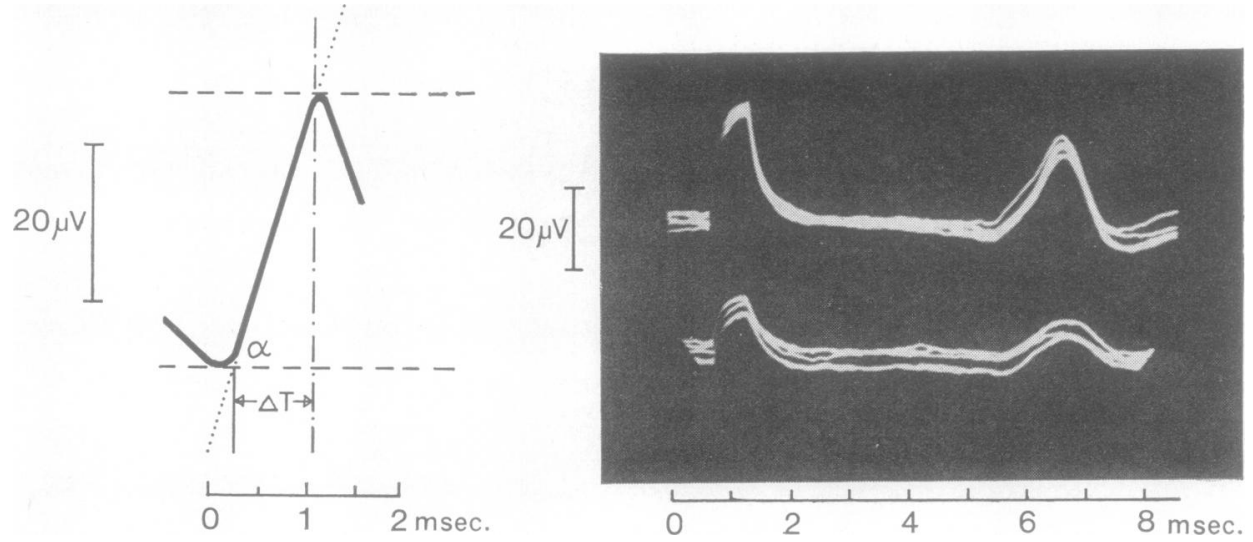

FIG. 1. Left: measurement of the steepness of ascending limb in the negative phase of nerve action potential (n. ulnaris), either as rise time of potential amplitude $(\triangle T)$, or as the tangent of the angle between the ascending limb of the negative phase and the isolectric line (tan $\alpha)$.

Right: stimulus dependence of rise time of the potential amplitude (n. ulnaris). Top record: nerve action potential at supramaximal stimulation $(110 \mathrm{~V})$. Bottom record: nerve action potential at near threshold stimulation $(50 \mathrm{~V})$. Percutaneous stimulation of the ulnar nerve above the wrist, nerve response recorded by surface electrodes at the elbow. In this and subsequent Figures upward deflection indicates negativity of the lower recording electrode with respect to the upper one. Normal subject (M.D., age 18).

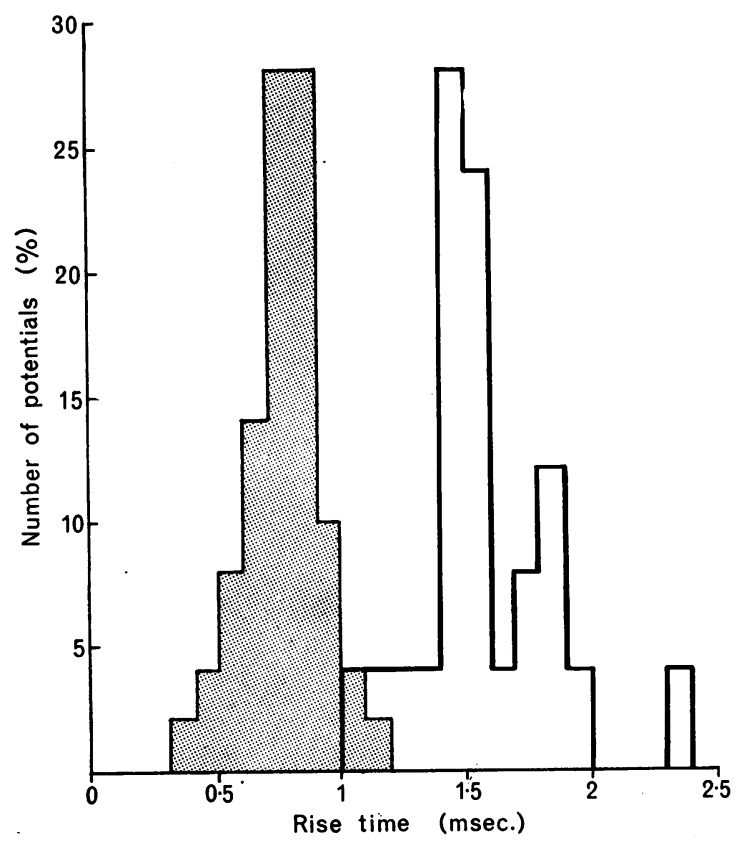

FIG. 2. Distribution of rise times of action potential amplitudes of the ulnar nerve at supramaximal stimulation. Shaded histogram: rise times in 50 healthy subjects. Unshaded histogram: rise times in a group of 25 patients with a slight lesion of peripheral nerve. Abscissa: Rise time (in milliseconds). Ordinate: Number of nerve action potentials (in percentage of occurrence).

\section{RESULTS}

In normal people the rise time histogram (Fig. 2) showed the peak distribution of rise times of the action potential amplitudes of the ulnar nerve at supramaximal stimulation at 0.65 to $0.85 \mathrm{msec}$. (with a mean of $0.728 \pm 0.022 \mathrm{msec}$.), whereas in patients with a peripheral nerve lesion the distribution of rise times was in marked contrast to this. There was a shift to the right, towards higher values, with a peak at 1.4-1.6 msec. (the mean value was $1.52 \pm 0.057 \mathrm{msec}$.). The distribution of rise times in control subjects is significantly different from that of patients with nerve lesions $(P<0.001)$.

The rise length $(\lambda)$ of the action potential in a damaged nerve was found to be prolonged as compared with that of a healthy one. For example (Fig. 3, A), distance (D) between the stimulating cathode at the wrist and lower recording electrode at the elbow is $28.0 \mathrm{~cm}$., latency (L) from onset of the stimulus artefact to the junction of the negative inflection potential and the baseline is $4.8 \mathrm{msec}$., and conduction velocity (v) in the healthy ulnar nerve is thus $v=\frac{D}{L}=\frac{28.0}{4.8}=5.83$, or $58.3 \mathrm{~m} . / \mathrm{sec}$.

If the rise time $(\triangle T)$ is $0.7 \mathrm{msec}$, the rise length $(\lambda)$ is then

$$
\begin{aligned}
\lambda & =\mathrm{v}(\triangle \mathrm{T}) \mathrm{mm} . \\
& =58 \cdot 3(0 \cdot 7) \mathrm{mm} . \\
& =40.81 \mathrm{~mm} . \\
& =4.081 \mathrm{~cm} .
\end{aligned}
$$




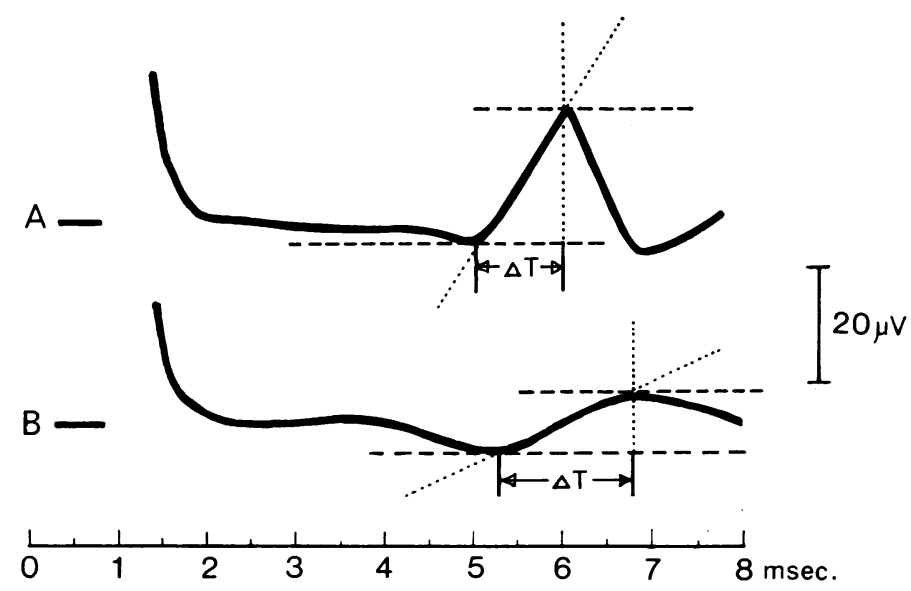

FIG. 3.
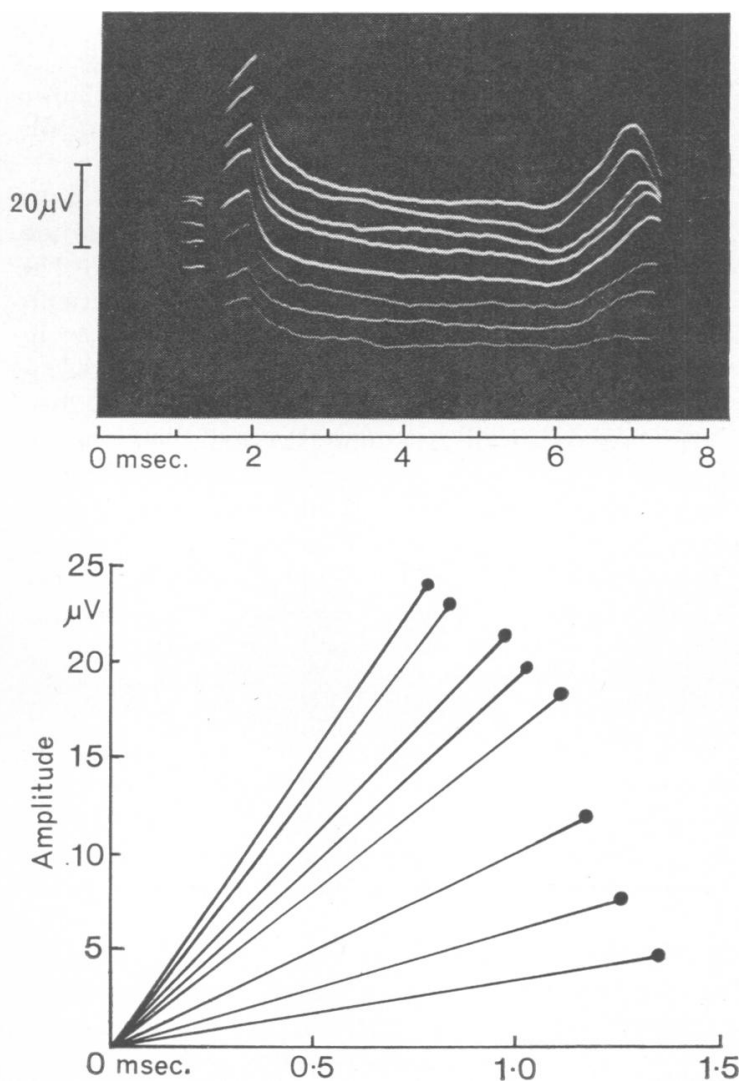

FIG. 4. or $6.97 \mathrm{~cm}$.
FIG. 3. Determination of rise length of action potential of the ulnar nerve. A-healthy subject (L.C., age 21). Bpatient with polyradiculoneuritis (K.F., age 40). Symbol $\triangle \mathrm{T}$ denotes the rise time.

FIG. 4. Effect of stimulus intensity on the rise time of nerve action potential ( $n$. ulnaris) in a healthy subject (M.D., age 18). Oscilloscopic tracings of nerve action potential (only the rising phase of potential amplitude is shown) at increasing intensity of stimulation (40, $50,60,70,80,90,100$, and $110 \mathrm{~V}$ ). Top trace, potential at $110 \mathrm{~V}$; bottom trace, potential at $40 \mathrm{~V}$. The diagram was plotted according to data obtained from the oscilloscopic tracings shown in the upper part of the figure. It shows changes of amplitude (ordinate) and rise time (abscissa) of the action potential at increasing stimulus intensity. The maximum point on each line is the peak of potential amplitude (spike). The topmost line represents the rising phase at supramaximal stimulation $(110 \mathrm{~V})$; the effect of near threshold stimulation (40 V) is shown by the bottom line.

In the damaged nerve (Fig. 3, B) conduction distance (D) is $23.0 \mathrm{~cm}$.. latency (L) is $5.6 \mathrm{msec}$. and conduction velocity $(\mathrm{v})$ is thus $\mathrm{v}=\frac{23.0 \times 10}{5.6}$,

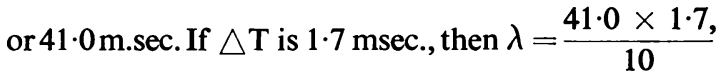

The relationship between stimulation intensity and time course of the action potential of an intact nerve is illustrated in Figure 4. In each of these experiments the nerve responses to electrical stimuli of progressively increasing intensity from near threshold to supramaximal and vice versa were recorded on the multiple-exposure photograph made by displacing the base line upwards or downwards after each change of stimulus strength. In Fig. 4 the rising phases of normal potentials recorded during a typical experiment are shown graphically. With decreasing strength of stimulation, the peak action potential amplitude becomes smaller and the rate of rise becomes slower. At near threshold stimulation, the effect of which is shown as the bottom line, the rise time attains the greatest value.

In peripheral nerve paresis, both of these effects become intensified: each decrease of stimulus strength, as in the experiment in Fig. 5, caused a greater decrease in rate of rise and lowering of 

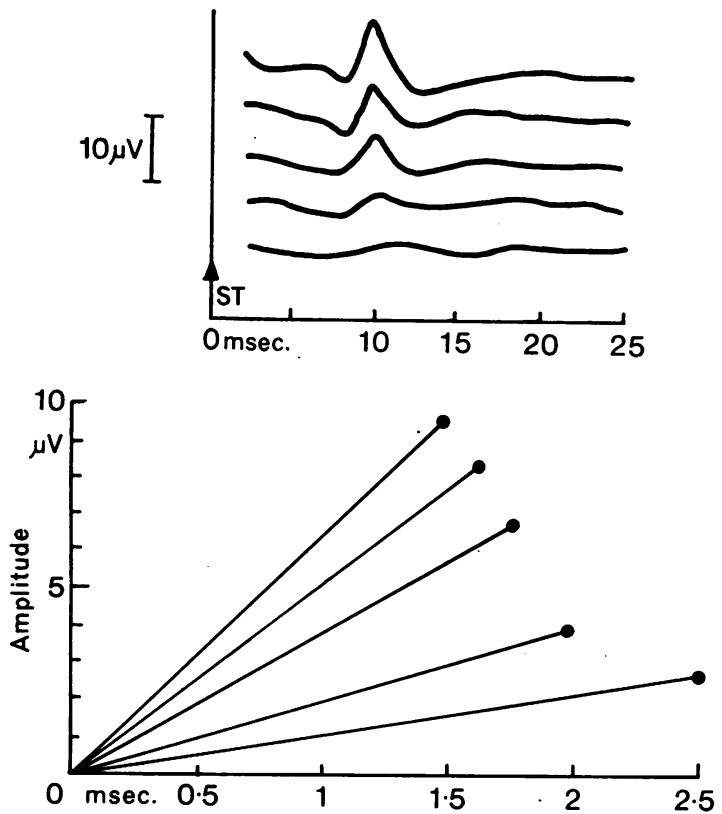

FIG. 5. Effect of stimulus intensity on the rise time of nerve action potential (n. ulnaris) in a patient with lesion of the peripheral nerve (K.F., age 40, with polyradiculoneuritis). The intensity of stimulation (from top to bottom) $110,100,90,80$, and $70 \mathrm{~V}$. Same indications as in Figure 4.

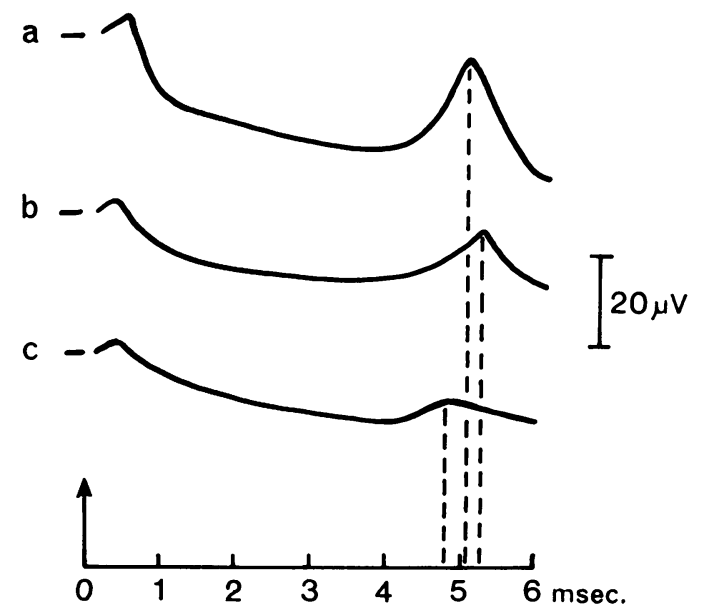

FIG. 6. Changes in various parameters of nerve action potential during graded strength of stimulation in a normal subject (H.P., age 38). The rise time of action potential increases with decreasing intensity of stimulation from supramaximal shock (a) to supraliminal (b). On the other hand the rise time declines with liminal stimulus intensity (c) although both amplitude and slope (tan $\alpha)$ of potential continue to diminish, and achieve their minimal value at threshold stimulation. action potential amplitude than in healthy individuals.

The curves of Fig. 4 and 5 show that amplitude and rise time change as a function of stimulus intensity. When the current pulse is extremely small, as in cases with threshold stimulation, sometimes some modification may occur. The rise time paradoxically becomes shorter although both the amplitude and the slope $(\tan \alpha)$ of potential decrease progressively, and attain their minimal value at an intensity of nerve stimulation which is just liminal (Fig. 6).

\section{DISCUSSION}

The main results of this investigation show that in normal subjects of different ages, the rise time $(\triangle T)$ for the nerve action potential evoked by weak stimulation is typically prolonged when compared with that attained at maximal and supramaximal intensity of nerve stimulation. In patients with peripheral neuropathy there was slowing of the rate of rise even at supramaximal shock strength when compared with the normal group, and decrease in rate of rise with decreasing intensity of stimulation also becomes more prominent here than in the normal subjects.

These results were not unexpected because some physical aspects of the stimulus parameters and their relations to the tissue are well established. The stimulus effect is mainly given by ionic movements in tissue and hence it is determined not only by the intensity of the field but also by its duration (time course). The surface membranes of the nerve fibre, both the myelin sheath and the nodal membrane, represent relatively large capacities. Therefore, higher stimulus intensities are needed at shorter stimulus durations to raise the membrane potential by a constant amount, e.g., to the threshold level (Tasaki, 1959). However, it is also well known that the current strength required for impulse conduction in axons and muscle fibres increases with decreasing rate of rise in current (Lucas, 1917; Katz, 1939). At near threshold stimulation, ionic currents displace not only the resting potential of the membrane of the axons to the threshold (critical) depolarization more slowly than in response to stronger shocks resulting in prolonged latency, but they also cause a very slow rate of rise so that the peak potential amplitude is attained after a relatively long period of time. Accordingly, modification of the membrane potential caused by a near threshold shock is very similar to that classically associated with long stimulating pulses as described by Tasaki (1939, 1959) and Knutsson (1964). Both in animals with experimental demyelination in peripheral nerves 
(Tasaki, 1955; McDonald, 1963a, b; Kaeser, 1963; Cragg and Thomas, 1964; Mayer and Denny-Brown, 1964) and patients with a mild nerve lesion (Bannister and Sears, 1962; Gilliatt and Willison, 1962; Mayer, 1963; Mawdsley and Mayer, 1965; Miglietta, 1966; and others) myelin destruction accompanied by relative preservation of axons was histologically demonstrated or clinically greatly suspected. The experimental results of Tasaki (1955) suggest that reduction in thickness of the myelin sheath increases its capacity and decreases its transverse resistance. This results in increased leakage of current through the damaged internodal myelin and will reduce the current density at the neighbouring nodes of Ranvier and thereby delay excitation at these still excitable nodes and slow down the reaching of the peak action current. The axon irregularities associated with some reduction in fibre diameter frequently seen in demyelinated nerve fibres will increase the longitudinal resistance of axons and thus contribute to the slowing of the rise in current at the nodes (Gasser and Grundfest, 1939; McDonald, 1963a, b). If the degree of the myelin disintegration is intensive and the length of such an abnormality very extensive, the membrane current then rises more slowly than a certain critical rate and fails to trigger conducted potentials. This phenomenon is closely related to the process of accommodation of the electrogenic membrane, in a large population of nerve fibres, to a slow, outwardly directed current from the area of the sink. Accordingly, such a membrane does not become depolarized to a critical level and blocking of conduction may occur in this pathological condition.

The pradoxical behaviour of the rise time at stimulus intensity which is just at the threshold level can only partly be due to fallacy in measurement, i.e., as a result of some difficulties in determining the exact point of the peak. This may be the case when potentials are too small, when the slope of their rise from the time base is very gradual and their top is rounded. Hodes, Gribetz, Moskowitz, and Wagman (1965) also gave a similar explanation for changes in peak latencies of muscle potentials at weak stimulation. But real changes in the rise time do exist, and may be explained on the basis of a strength-duration relation. When the duration of the current pulse becomes far shorter or longer than the time constant of the resting axon membrane, the phenomenon of abolition of an action potential occurs (Tasaki, 1959). In the case of a threshold stimulation, the current pulse is extremely small, and the uncharged membrane on both sides of the excitation locus may prevent a further rise in potential amplitude at the locus of excitation, e.g., under the lower (active) recording electrode, so that the peak level of such a potential is reached prematurely within a shortened period of time.

However, experiments have not yet been done on the relationship between rate of rise of the nerve action potential and the stimulus intensity in man. Hence, our discussion and conclusions concerning the stimulus dependence of the rate of rise can only be preliminary and tentative. On the basis of our experiments, we may assume that the slope of the potential rise is very sensitive to reduction of stimulus intensity and that this is especially pronounced in cases of peripheral neuropathy.

\section{SUMMARY}

Rise times of the negative (main) deflection of triphasic nerve response $(\triangle T)$ at threshold and at supramaximal stimulation of the ulnar nerve were studied in 50 healthy subjects and in 25 patients with nerve lesions. The nerve was stimulated at the wrist and the response recorded above the elbow.

Rise times in patients with peripheral nerve lesions were markedly prolonged as compared with those in control subjects (mean: 0.728 $\pm 0.022 \mathrm{msec}$. in controls; $1.52 \pm 0.057 \mathrm{msec}$. in patients).

The distribution of rise times in control subjects was significantly different from that of patients with nerve lesions $(\mathrm{P}<0.001)$.

Accordingly, the rise length $(\lambda)$ of the action potential in a damaged nerve was found to be characteristically prolonged in comparison with that of a healthy one.

With decreasing strength of stimulation, rise times become longer and reach the greatest value at near threshold stimulation.

In peripheral nerve paresis, this effect becomes much more prominent than in healthy invididuals.

The results involving the effect of stimulus intensity on rate of rise of the action potential suggest a marked sensitivity of the slope of rise to reduction of stimulus intensity, mainly in cases of peripheral neuropathy. Thus, changes in rate of rise provide a highly sensitive index of peripheral nerve damage and would be useful in detecting the early occurrence of a nerve lesion.

The author wishes to express his appreciation to Mrs. Pafková for her excellent assistance, to Ing. G. Hudáková for her advice on the statistics, and to the Medical Photography and Film Institute for the careful photography.

\section{REFERENCES}

Bannister, R. G., and Sears, T. A. (1962). The changes in nerve conduction in acute idiopathic polyneuritis. J. Neurol. Neurosurg. Psychiat., 25, 321-328.

Bureš, J., Petrán̆, M., and Zachar, J. (1960). Electrophysiological Methods in Biological Research, pp. 276-280. Publishing House of the Czechoslovak Academy of Sciences, Prague. 
Cragg, B. G., and Thomas, P. K. (1964). Changes in nerve conduction in experimental allergic neuritis. J. Neurol. Neurosurg. Psychiat., 27, 106-115.

Dawson, G. D., and Scott, J. W. (1949). The recording of nerve action potentials through skin in man. Ibid., 12, 259-267.

Gasser, H. S., and Grundfest, H. (1939). Axon diameters in relation to the spike dimensions and the conduction velocity in marrmalian A fibers. Amer. J. Physiol., 127, 393-414.

Gilliatt, R. W., and Sears, T. A. (1958). Sensory nerve action potentials in patients with peripheral nerve lesions. J. Neurol. Neurosurg. Psychiat., 21, 109-118.

_- and Willison, R. G. (1962). Peripheral nerve conduction in diabetic neuropathy. Ibid., 25, 11-18.

Hodes, R., Gribetz, I., Moskowitz, J. A., and Wagman, I. H. (1965). Low threshold associated with slow conduction velocity. Arch. Neurol. (Chic.), 12, 510-526.

Kaeser, H. E. (1963). Die experimentelle diphtherische Polyneuritis. Karger, Basel and New York. (Bibliotheca Psychiat. neurol., fasc. 120.)

Katz, B. (1939). Electric Excitation of Nerve. Oxford University Press, London.

Knutsson, E. (1964). Subthreshold depolarizations in skeletal muscle fiber during linearly rising current. Acta physiol. scand., 61, Suppl. 224.

Kostyuk, P. G. (1956). About the site of the origin of electrotonic potentials in spinal roots at the stimulation of muscle nerves. (In Russian), Fiziol. Zh. (Moscow), 42, 800-810.

Lloyd, D. P. C. (1952). Electrical manifestations of action in neurons. In The Biology of Mental Health and Disease: the Twentyseventh Annual Conference of the Milbank Memorial Fund, New York, 1950, pp. 135-161. Hoeber, New York.

Lorente de Nó, R. (1947a, b). A Study of Nerve Physiology. (Stud. Rockefeller Inst. med. Res., Vols. 131-132.)

Lucas, K. (1917). The Conduction of the Nervous Impulse, rev. E. D. Adrian. Longmans, London.
McDonald, W. I. (1963a). The effects of experimental demyelination on conduction in peripheral nerve: A histological and electrophysiological study. I. Clinical and histological observations. Brain, 86, 481-500.

- (1963b). The effects of experimental demyelination on conduction in peripheral nerve: $\mathbf{A}$ histological and electrophysiological study. II. Electrophysiological observations. Brain, 86, 501-524.

Mawdsley, C., and Mayer, R. F. (1965). Nerve conduction in alcoholic polyneuropathy. Brain, 88, 335-356.

Mayer, R. F. (1963). Nerve conduction studies in man. Neurology (Minneap.), 13, 1021-1030.

, and Denny-Brown, D. (1964). Conduction velocity in peripheral nerve during experimental demyelination in the cat. Ibid., 14, 714-726.

Miglietta, O. (1966). Nerve motor fiber characteristics in chronic ischemia. Arch. Neurol. (Chic.), 14, 448-453.

Paintal, A. S. (1966). The influence of diameter of medullated nerve fibres of cats on the rising and falling phases of the spike and its recovery. J. Physiol. (Lond.), 184, 791-811.

Podivinský, F. (1965a). The effect of stimulus intensity on the amplitude of nerve action potentials in healthy subjects and in patients with peripheral nerve lesions (in Slovakian). Bratisl. Lek. Listy, 45, (I), 330-337.

- (1965b). The effect of stimulus intensity on latency of nerve action potential in healthy subjects and in patients with peripheral nerve lesions. Neurology (Minneap.), 15, 1059-1062.

Tasaki, I. (1939). The strength-duration relation of the normal, polarized and narcotized nerve fiber. Amer. J. Physiol., 125, 367-379.

- (1955). New measurements of the capacity and the resistance of myelin sheath and the nodal membrane of the isolated frog nerve fiber. Ibid, 181, 639-650.

- (1959). Conduction of the nerve impulse. In Handbook of Physiology, Section 1: Neurophysiology, vol. I, pp. 94-98, and 100-102. American Physiological Society, Washington, D.C. 